\title{
Metal-Free Colorimetric Detection of Pyrophosphate Ions by Inhibitive Nanozymatic Carbon Dots
}

Chong-You Chen, " Ying Zi Tan, Ping-Hsuan Hsieh, Chang-Ming Wang, 'Hiroyuki Shibata,

Kento Maejima, ${ }^{,}$Ting-Yi Wang, ${ }^{\prime}$ Yuki Hiruta, ${ }^{\S}$ Daniel Citterio,,${ }^{,} *$ Wei-Ssu Liao", *

$\dagger$ Department of Chemistry, National Taiwan University, Taipei 10617, Taiwan

\$ Material and Chemical Research Laboratories, Industrial Technology Research Institute, Hsinchu 30011, Taiwan

$\S$ Department of Applied Chemistry, Keio University, Yokohama 223-8522, Japan

*To whom correspondence should be addressed:

citterio@applc.keio.ac.jp; wsliaochem@ntu.edu.tw 
(A)

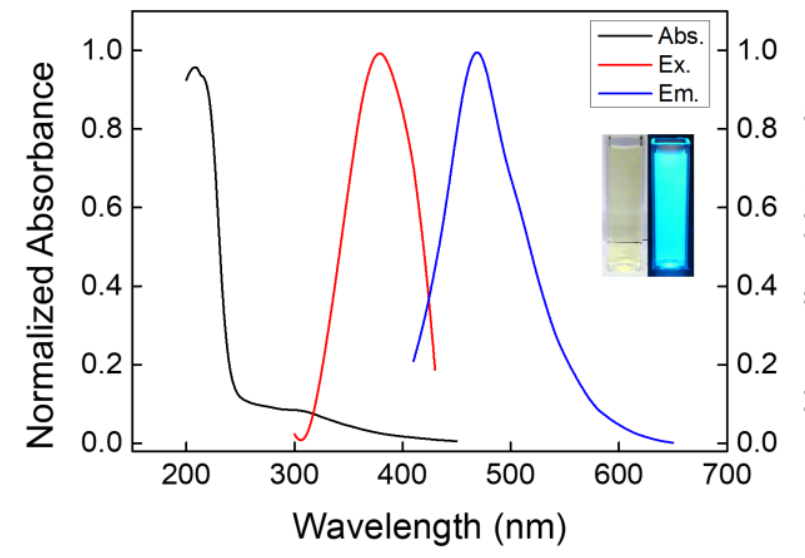

(B)

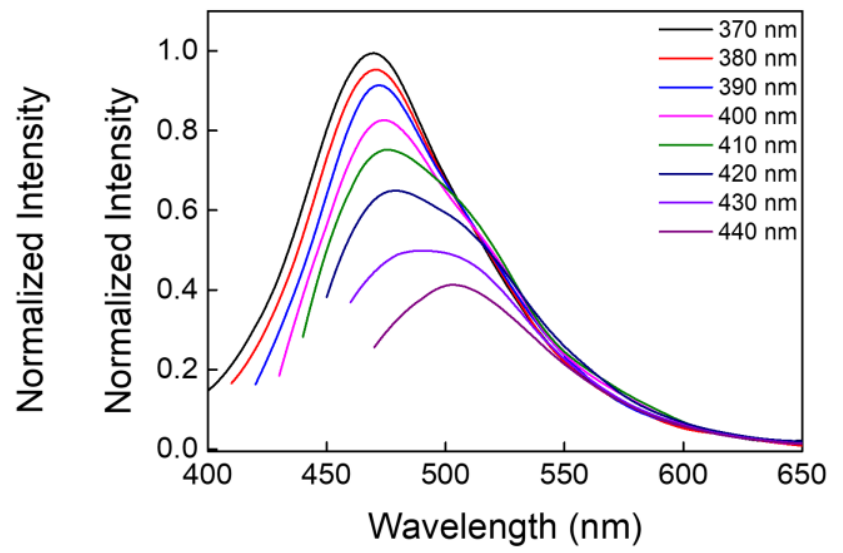

Figure S1. Optical properties of the synthesized CDs. (A) Overlapping UV-Vis absorption and fluorescence excitation/emission spectra of CDs. Inset: photographs of the CDs solution under white light (left) and $365 \mathrm{~nm}$ of UV illumination (right). (B) Fluorescence emission spectra of CDs under different wavelength of excitation. 
(A)

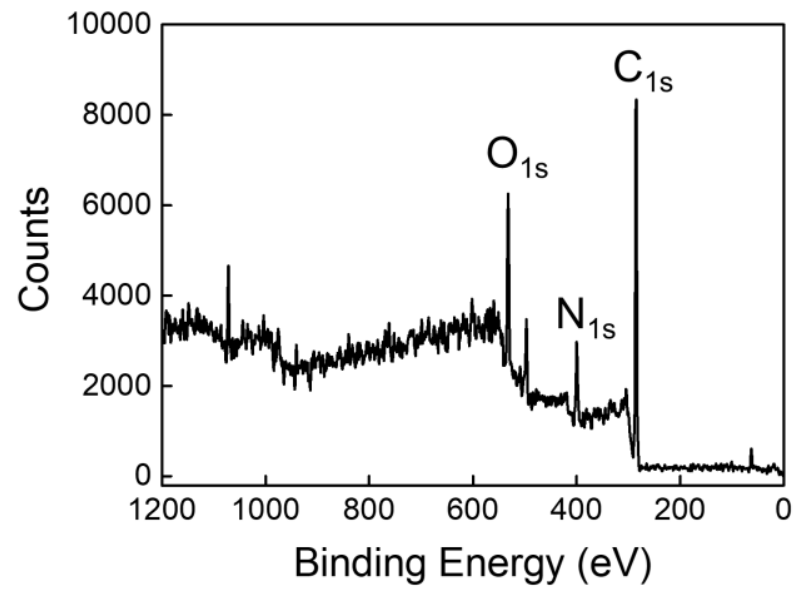

(C)

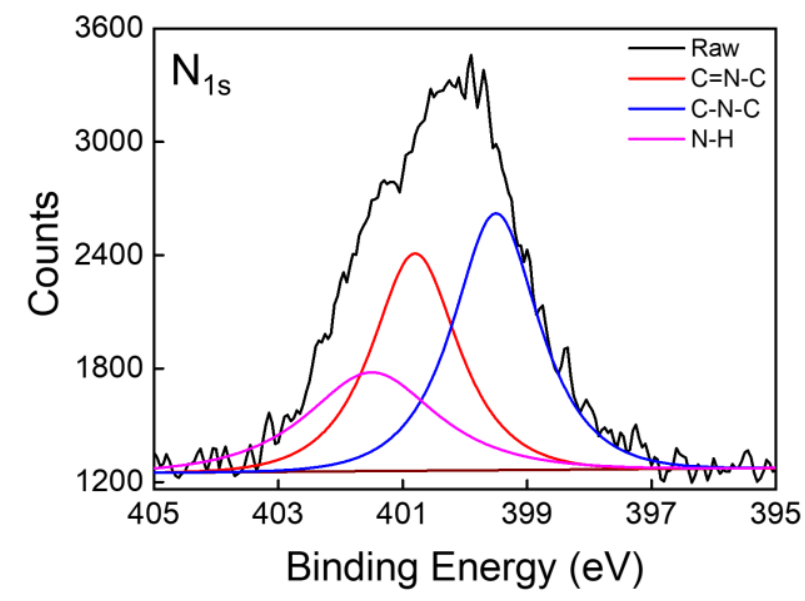

(B)

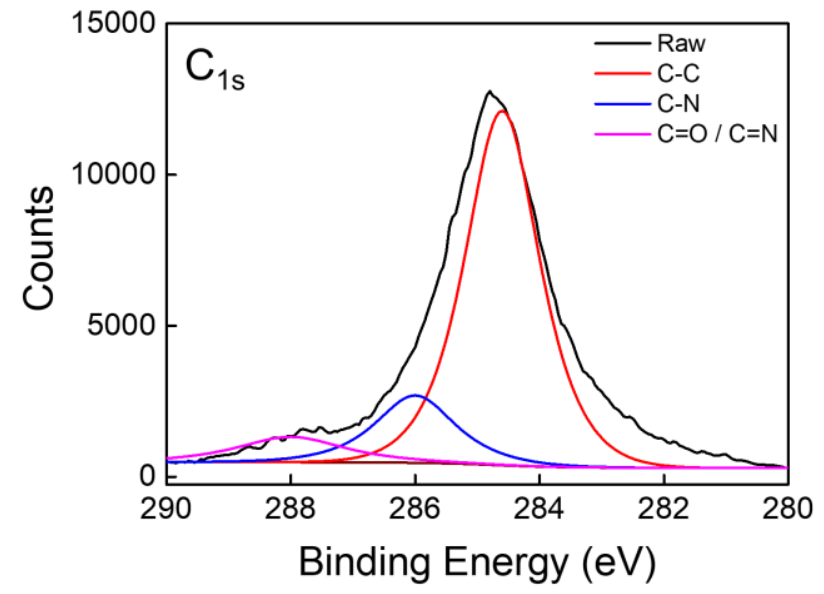

(D)

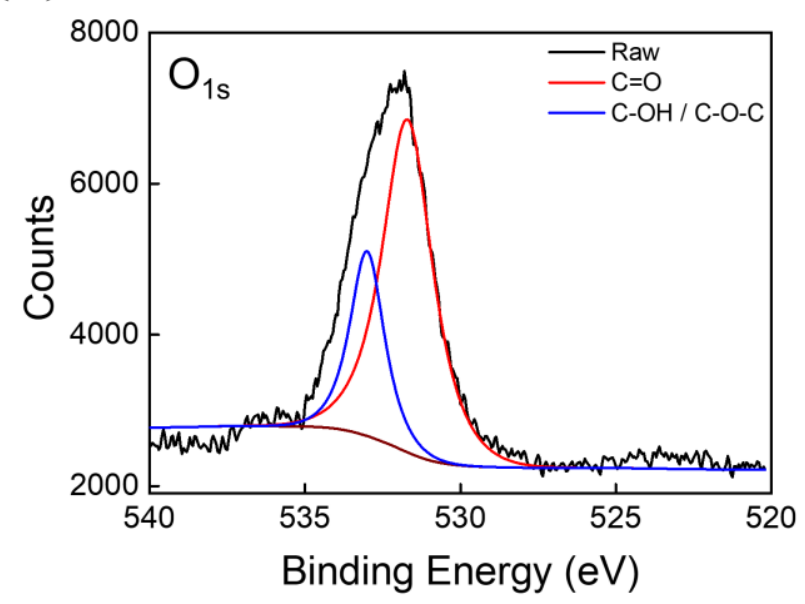

Figure S2. XPS spectra of synthesized nanozymatic CDs. (A) Wide scan; (B) C 1s core-level; (C) N 1s core-level; (D) O 1s core-level. 


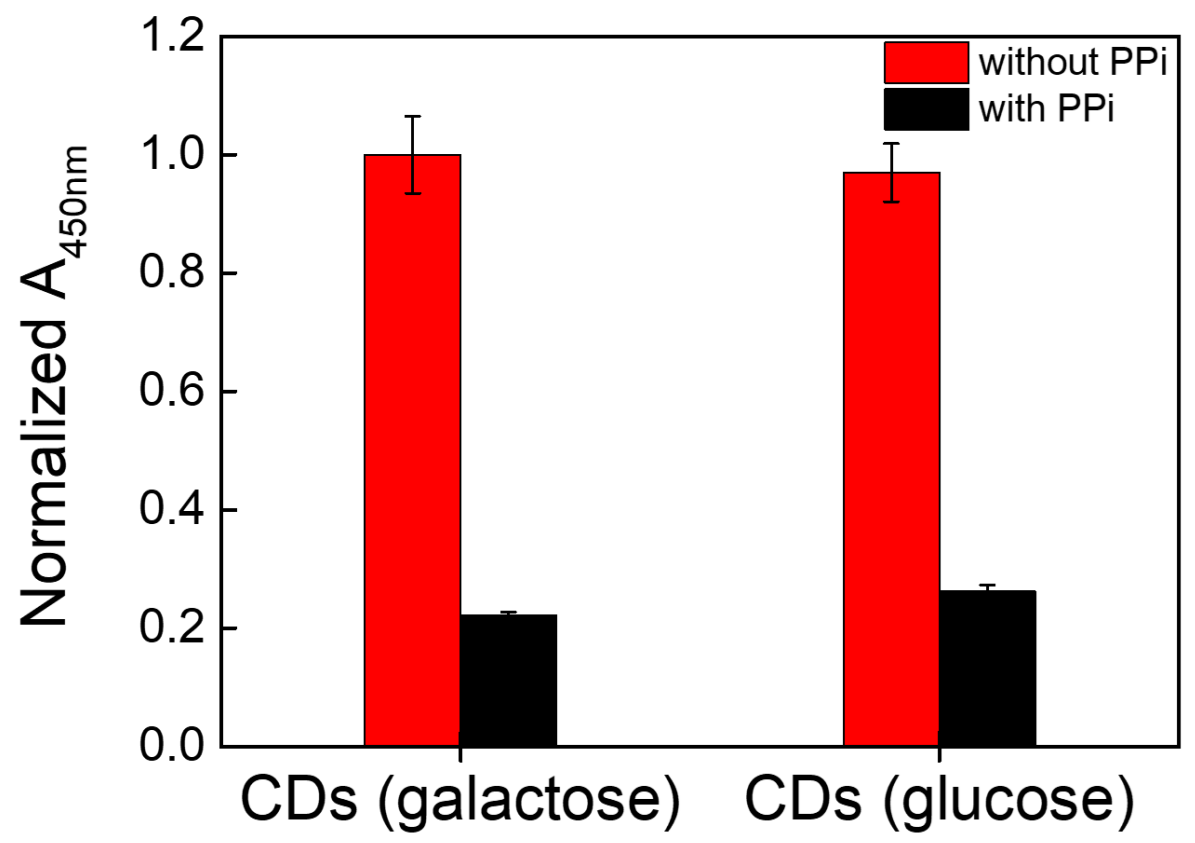

Figure S3. Catalytic OPD oxidation using CDs prepared from galactose or glucose precursors (both with histidine molecules), and their corresponding PPi inhibition performance. The error bars indicate the standard deviation. $(N=3)$ 


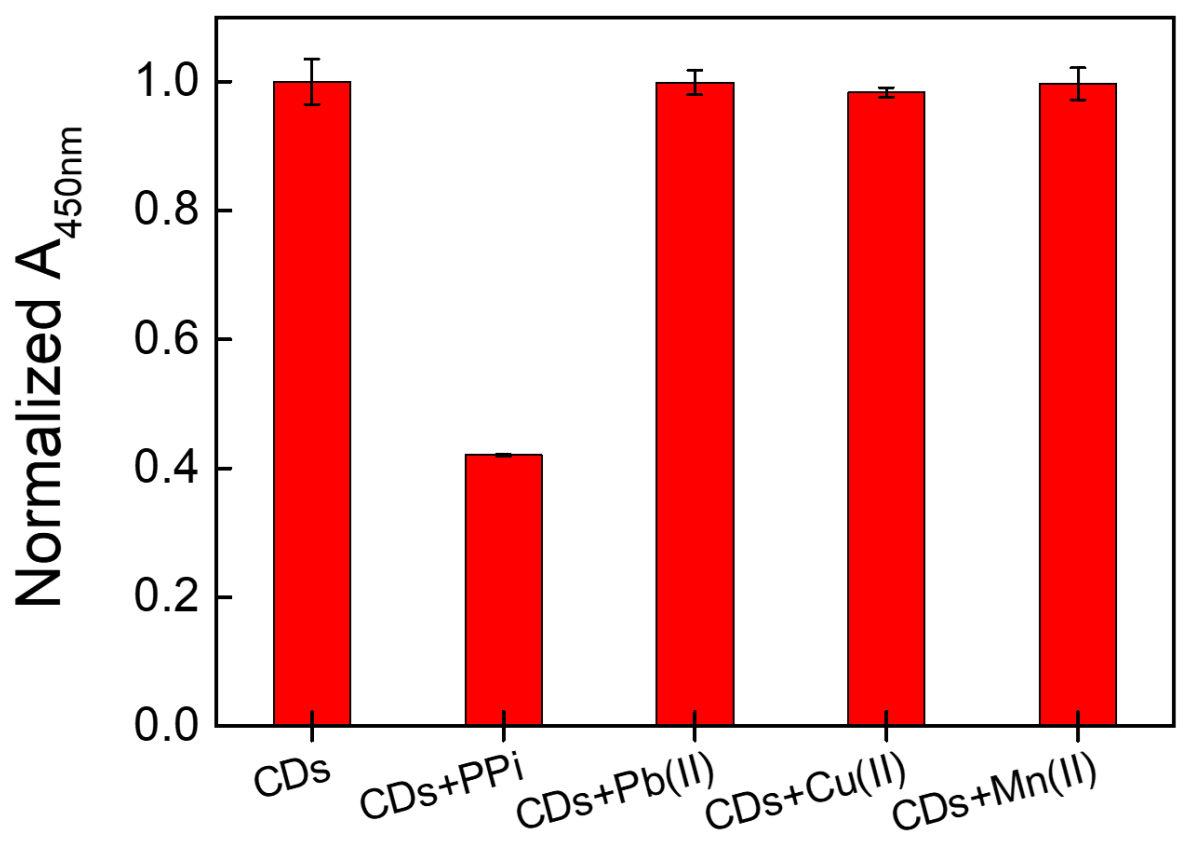

Figure S4. Influences of different metal ions $(1 \mu \mathrm{M})$ on the catalytic OPD oxidation inhibition. The error bars indicate the standard deviation. $(N=3)$ 


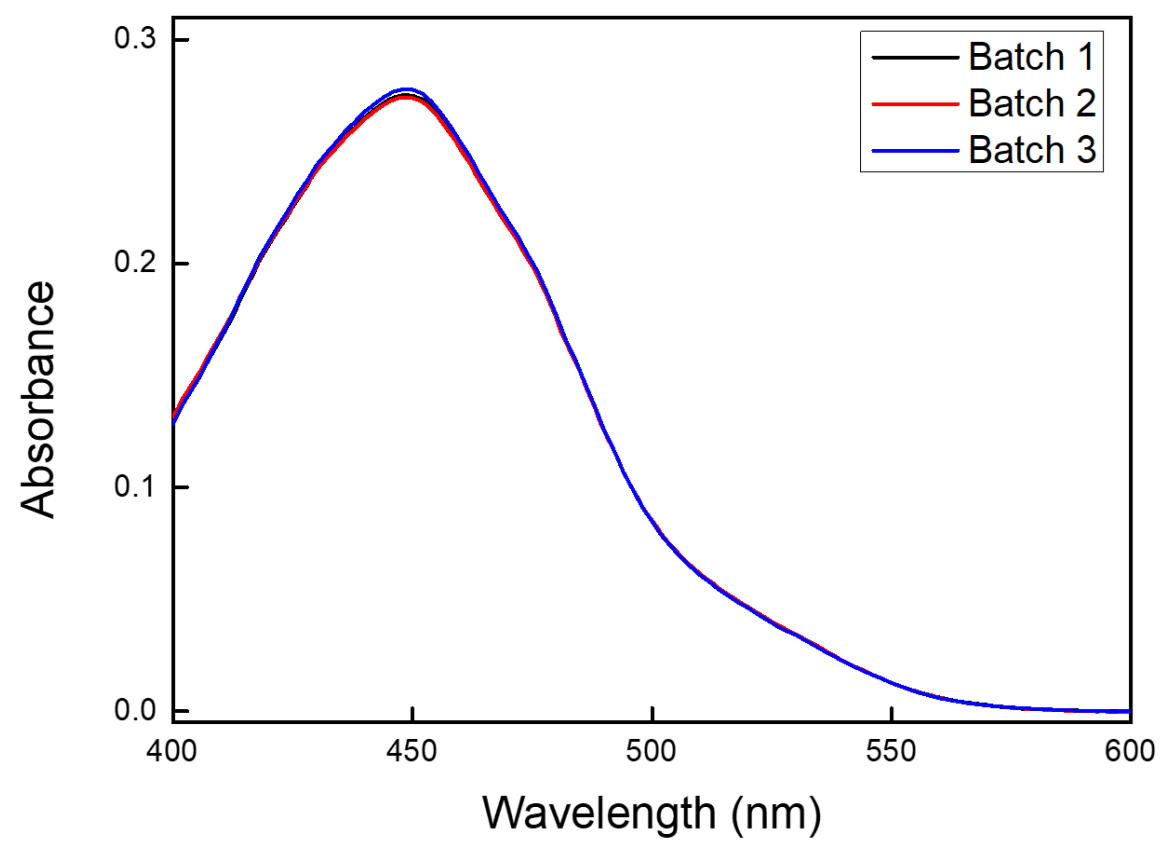

Figure S5. The catalytic OPD oxidation in the presence of $\mathrm{H}_{2} \mathrm{O}_{2}$, triggered by enzymatic CDs prepared from different batches. Similar DAP characteristic peaks at $450 \mathrm{~nm}$ indicate the high consistency of synthesized CDs. 